\title{
Proposta para Avaliação Quantitativa para Aquisição de Equipamentos Médicos
}

\author{
Proposal for Quantitative Evaluation for Medical \\ Equipment Acquisition
}

Marcela Cristina Chaves Penco ${ }^{1}$; Ernesto Fernando Ferreyra Ramírez ${ }^{2}$

Resumo

Este artigo apresenta uma proposta para estimar o número ideal de equipamentos médicos a ser comprado por uma instituição de saúde. O número de equipamentos necessários ("n") no setor avaliado, pode ser calculado a partir de uma equação, na qual são considerados os seguintes parâmetros: número de leitos ou usuários (" $\mathrm{n}_{\mathrm{b}}$ "); número de equipamentos existentes ("m"); proporção recomendada de equipamentos por paciente ("rr" número sugerido pelo Ministério da Saúde); importância do equipamento no cuidado do paciente ("v" pode ser suporte à vida, melhora no atendimento ao paciente ou desejável); e a condição física dos equipamentos existentes ("c" dados obtidos a partir dos relatórios de manutenção). Estes dados foram obtidos a partir de relatórios de manutenção preventiva dos equipamentos do Hospital Universitário da Universidade Estadual de Londrina (HURNP/UEL). Foram considerados dois estudos de caso: no primeiro caso, o método foi aplicado a bombas infusoras em dois diferentes setores do hospital (UTI e enfermarias). No segundo estudo de caso foram comparadas as quantidades ideais de diferentes equipamentos (oxímetro de pulso, oxicapnógrafo e monitor multiparamétrico) que possuam a mesma função (oximetria), em um único setor (centro cirúrgico). Apesar de sugerirem a necessidade de alguns aperfeiçoamentos na metodologia, os estudos de caso mostraram que a mesma é de fácil aplicação e pode fornecer uma idéia inicial das necessidades clínicas dos equipamentos médicos dos diversos setores de um hospital. Em alguns, casos outros fatores devem ser analisados como o setor onde o equipamento está e a possibilidade de existirem outros equipamentos com a mesma função. Além disso, este artigo mostra a necessidade de um método de priorização entre setores no hospital, fator importante na avaliação dos locais que realmente necessitam de reposição de equipamentos.

Palavras-chave: Engenharia clínica; Processo de aquisição de equipamentos médicos; Avaliação quantitativa.

\footnotetext{
${ }^{1}$ Marcela Cristina Chaves Penco - Acadêmica do Curso de Engenharia Elétrica, Centro de Tecnologia e UrbanismoUEL.Email:marcelapenco@sercomtel.com.br

2 Prof. M.Sc. do Departamento de Engenharia Elétrica, Centro de Tecnologia e Urbanismo-UEL.Email:ferreyra@uel.br
} 


\begin{abstract}
This paper proposes a method to evaluate the ideal number of medical equipment to be acquired by a Brazilian healthcare institution. The number of equipment needed (" $n$ ") in a hospital sector can be calculated with one expression, where the following parameters are taken into consideration: number of beds or users ("nb"); number of existing equipment ("m"); recommended rate of equipment per patient ("rr" that was obtained from Brazilian standard published by the Health Ministry); equipment importance to patient care (" $\mathrm{v}$ " that could be: life-support, improvement of the service or advisable); and the equipment condition ("c" that results from maintenance history data). These variables were obtained with data gathered from database and medical equipment preventive maintenance reports of the University Hospital of Londrina - Paraná - Brazil (HURNP/UEL). Two case studies were carried out. In the first case, the method was applied to work out the ideal number of infusion pumps needed for two different sectors (ICU and Nurseries) in the hospital. The second study compared the ideal quantities of different equipment (pulse oximeter, oxicapnograph and multiparameter monitor) that offered the same function (oximetry), in a surgical center. These results showed that this methodology is simple and easy to apply, although there must be an improvement of some aspects such as the local where the equipment is working and the possibility of different equipment performing the same function. Additionally, this paper showed the importance of implementing a prioritizing method among hospital sectors, because it will help to allocate resources where they are really necessary.
\end{abstract}

Key words: Clinical Engineering; Acquisition Process; Quantitative Evaluation.

\section{Introdução}

Estudos mostram que uma porção significativa dos equipamentos médico-hospitalares nos países em desenvolvimento está fora de uso (LEE, 1995). No Brasil, estes números estavam entre $20 \%$ e $40 \%$ do parque instalado de equipamentos médicos instalados (WANG; CALIL, 1991), o que, em números atuais isto representaria um desperdício de US\$260 milhões a US\$ 520 milhões, em um mercado estimado em US\$ 1,3 bilhão/ano (CALIL, 2001). A falta de planejamento adequado no momento da aquisição destes equipamentos foi um dos fatores que levaram a isto, por isso, a elaboração adequada da especificação técnica do equipamento é um ponto importante no planejamento e aquisição de equipamentos. Além disso, a falta de um dimensionamento adequado do serviço leva aos mesmos problemas mencionados anteriormente (MULLER JR.; CALIL, 2000). Assim se mostra necessária uma avaliação tanto das necessidades reais da instituição quanto do equipamento em questão. Eis a importância do trabalho do engenheiro clínico, um profissional não puramente técnico e científico, mas que interage com a equipe clínica e toma a liderança em todo processo de avaliação de equipamentos e tecnologias, visando à melhoria no atendimento ao paciente e levando em conta os interesses da instituição (CAPUANO, 1997).

O processo de avaliação de equipamentos, apesar de envolver a decisão humana, pode tornar-se um método científico, independente da opinião individual dos avaliadores (STIEFEL; RISKALLA, 1995; RAMIREZ; JERONYMO NETO; JANNANI, 2001). Dentro desta linha temos o trabalho de Tawfik (1994), que propõe um método para determinar o número de equipamentos necessários por meio de um modelo algébrico. Utilizando-se de questionários direcionados aos departamentos envolvidos e testes com situações hipotéticas, este método gera um guia que ajuda a determinar as quantidades mais adequadas a serem oferecidas para o departamento. Mais recentemente, Müller Jr. e Calil (2000) apresentaram um sistema computacional que dá suporte para tomadas de decisões de modo a facilitar a seleção e a especificação de equipamentos médicos e auxilia no planejamento de serviços de saúde, oferecendo ao usuário a quantidade e a especificação de equipamentos, móveis, e objetos necessários.

Assim, este trabalho propõe um método para estimar o número de equipamentos médicos que 
devem ser adquiridos para suprir as necessidades estabelecidas pelos usuários de um E.A.S. (Estabelecimento Assistencial de Saúde).

\section{Metodologia}

A metodologia proposta é basicamente a aplicação da equação elaborada por Tawfik (1994), com algumas alterações, num sistema computacional baseado na proposta de Müller Jr. e Calil (2000).

A equação proposta por Tawfik (1994) é, originalmente:

$\mathrm{n}=\operatorname{INT}\left(\mathrm{n}_{\mathrm{b}} \cdot \operatorname{rr} . \mathrm{v} \cdot \mathrm{g}\right)-\operatorname{INT}\left(\sum_{i=1}^{m} c_{i}\right)$

onde:

- INT é a parte inteira do número resultante da somatória indicada;

- "n"= número de equipamentos necessários;

- "n $\mathrm{n}_{\mathrm{b}}$ "= número de leitos ou usuários;

- "rr"= proporção recomendada de equipamento por paciente;

- "m"= número existente de equipamentos;

- "c"= condição do equipamento;

- "v"= valor do equipamento no cuidado do paciente;

- "g"= capacidade de gerar lucros do equipamento.

\section{Determinação dos parâmetros}

A proporção recomendada de equipamento por paciente ("rr") foi determinada a partir das normas publicadas pelo Ministério da Saúde (1994). Os valores de "rr" para as simulações feitas são as mostradas nas Tabelas 1 e 2, a seguir:

Tabela 1 - Valor do "rr" para bombas infusoras

\begin{tabular}{l|c}
\hline \multicolumn{1}{c|}{ Setor } & "rr" \\
\hline UTI & 1 \\
\hline Enfermaria & 0.1 \\
\hline
\end{tabular}

Tabela 2 - Valores de "rr" para o Centro Cirúrgico

\begin{tabular}{l|c}
\hline \multicolumn{1}{c|}{ Equipamento } & "rr" \\
\hline Oxímetro de pulso & 1 \\
\hline Oxicapnógrafo & 1 \\
\hline Monitor Multiparamétrico & 1 \\
\hline
\end{tabular}

Os valores da condição do equipamento ("c") foram atribuídos de acordo com os dados resultantes do estudo dos relatórios de manutenção dos equipamentos. Para a simulação feita, foram considerados com manutenção regular $(\mathrm{c}=1 / 2)$ todos os equipamentos que foram reprovados nos testes feitos, ou seja, os equipamentos que se encontravam descalibrados e para os equipamentos com manutenção $\mathrm{OK}$, ou seja, funcionando corretamente foi atribuído valor " 1 ".

$\mathrm{O}$ valor do equipamento no cuidado do paciente é classificado da seguinte forma:

- Suporte à vida: aparelhos que substituem temporariamente funções do organismo $(\mathrm{v}=1)$,

- Melhoria à qualidade do atendimento: equipamentos relacionados à prestação de serviço, sem os quais dificulta-se a prestação do mesmo $(v=3 / 4)$,

- Recomendável: equipamentos relacionados à prestação de serviço, sem os quais o mesmo pode ser prestado, só que em condições diminuídas de conforto e facilidade $(\mathrm{v}=1 / 2)$.

À variável que indica a capacidade de gerar lucros do equipamento atribui-se o valor " 1 " em todos os casos. Podemos fazer isso sem comprometer a avaliação, porque a variável "g" não tem grande influência no valor final, já que multiplica o resultado por " 1 "ou " 1,1 ". Além disso, somente a determinação deste item já seria tema para um outro trabalho, como o realizado por Katz (1998), que avaliou o custo do ciclo de vida de equipamentos médicos em hospitais. No caso de uma avaliação mais específica, em que a lucratividade não seja somente um "critério de desempate" entre dois equipamentos equivalentes, seria interessante a inserção deste item na análise. 
Depois de determinar todos os parâmetros utilizados para o cálculo do número de equipamentos necessários, foi montada uma planilha de cálculo para simular situações envolvendo diferentes setores de um hospital. Os dados utilizados para estas simulações (número de leitos, de equipamentos e condições dos mesmos) foram obtidos dos relatórios de trabalhos e dos artigos publicados (FILIZARDO; ITANO; RAMÍREZ, 2002; HIRAMA et al., 2002) relatando a experiência de elaboração de rotinas de manutenção preventiva para os equipamentos do Hospital Universitário da UEL (HURNP/UEL), por docentes e alunos do curso de Engenharia Elétrica da mesma instituição de ensino.

\section{Resultados}

Para os estudos de caso a seguir, os dados apresentados foram inseridos numa planilha de cálculo que determinou o número de equipamentos necessários a partir da equação 2 .

\section{- Estudo de caso \# 1- Bombas infusoras:}

O primeiro estudo de caso foi a aplicação da metodologia para as bombas infusoras de dois setores distintos do HURNP/UEL (UTI e Enfermaria). Os resultados são mostrados na Tabela 3 .

Tabela 3 - Simulação para bombas infusoras na UTI e Enfermaria do HURNP/UEL

\begin{tabular}{l|c|r}
\hline Equipamento & \multicolumn{2}{|c}{ Bombas Infusoras } \\
\hline Setor & UTI & Enfern \\
\hline $\mathrm{n}_{\mathrm{b}}$ & 17 & 13 \\
\hline $\mathrm{rr}$ & 1 & 0,1 \\
\hline $\mathrm{v}$ & 1 & 4 \\
\hline $\mathrm{c}$ (equipamentos OK) & 9 & 1 \\
\hline $\mathrm{c}$ (equipamentos com defeito) & 3 & 0 \\
\hline $\mathrm{c}$ (pouca manutenção) & 0 & 3 \\
\hline $\mathrm{c}$ (manutenção regular) & 10 & 1 \\
\hline $\mathrm{c}$ (manutenção freqüente) & 0 & $\mathbf{8}$ \\
\hline $\mathbf{n}$ & $\mathbf{3}$ & \\
\hline
\end{tabular}

\section{- Estudo de caso \# 2 - Oxímetros de Pulso:}

Este estudo de caso consiste em 4 partes. As três primeiras são estudos de oxímetros de pulso, oxicapnógrafos e monitores multiparamétricos separadamente. Visto que estes três equipamentos possuem a função de oximetria, também se fez uma simulação com o número total de equipamentos que possuem esta função, cujos resultados são apresentados na última coluna da Tabela 4. As quatro simulações foram feitas para equipamentos do Centro Cirúrgico do HURNP/UEL. 
Tabela 4 - Simulação para oxímetros de pulso, oxicapnógrafos e monitores multiparamétricos do Centro Cirúrgico do HURNP/UEL

\begin{tabular}{l|c|c|c|c}
\hline Equipamento & Oxímetro de pulso & Oxicapnógrafo & Monitor Multiparamétrico & Todos os ' \\
\hline $\mathrm{n}_{\mathrm{b}}$ & 14 & 14 & 14 & \\
\hline $\mathrm{rr}$ & 1 & 1 & 1 & \\
\hline $\mathrm{v}$ & 1 & 1 & 6 & \\
\hline $\mathrm{c} \mathrm{(equipamentos} \mathrm{OK)}$ & 3 & 5 & 0 & \\
\hline $\mathrm{c}$ (equipamentos com defeito) & 2 & 1 & 0 & \\
\hline $\mathrm{c} \mathrm{(pouca} \mathrm{manutenção)}$ & 0 & 0 & 0 & \\
\hline $\mathrm{c}$ (manutenção regular) & 0 & 0 & $\mathbf{8}$ & \\
\hline $\mathrm{c} \mathrm{(manutenção} \mathrm{freqüente)}$ & 0 & 0 & $\mathbf{9}$ & \\
\hline \multicolumn{1}{c}{$\mathbf{N}$} & $\mathbf{1 1}$ & &
\end{tabular}

\section{Discussão}

No primeiro estudo de caso foi simulada necessidade de um determinado equipamento para diferentes setores do hospital. Na tabela 3, são mostrados os resultados de tais simulações. Para a UTI, seriam necessárias 3 bombas infusoras para que o setor funcionasse sempre com 1 equipamento por paciente, ao passo que na Enfermaria esse número seria 8. É interessante a análise dessa situação, pois a aquisição de 3 bombas infusoras para a Enfermaria atenderia um número maior de pacientes, porém os pacientes internados na UTI têm maior necessidade de tais equipamentos. Um estudo de prioridades entre os setores ajudaria a definir o que fazer em tal situação. A UTI tem prioridade sobre a Enfermaria, pois os casos mais graves, que geralmente envolvem risco à vida do paciente, estão neste setor do hospital, enquanto que na Enfermaria nem todos pacientes necessitam do uso de uma bomba infusora.

No segundo estudo de caso a simulação foi realizada para equipamentos diferentes, que possuem uma mesma função, num mesmo setor do hospital. Comparando-se as quatro simulações, pode-se perceber não ser possível fazer um estudo que se limite determinar os equipamentos por nome. Por meio de um estudo ampliado, no qual são avaliados todos os equipamentos que possuem a mesma função (neste caso, todos os equipamentos que possuem a função de oximetria) tem-se uma visão diferente da situação do setor. A Tabela 4 mostra as simulações para oxímetros de pulso, oxicapnógrafos e monitores multiparamétricos separadamente e a última coluna mostra os resultados de uma simulação na qual todos os equipamentos que possuem a função de oximetria são incluídos. No caso da análise dos equipamentos em separado tem-se um resultado desfavorável, indicando a necessidade de aquisição de mais equipamentos para um funcionamento do setor sempre com um equipamento de oximetria por paciente. Porém, no segundo caso, o resultado é um número negativo, que indica que existem equipamentos em número maior que o mínimo para o funcionamento do setor nas condições já mencionadas. Entretanto, não foi investigado se essa redundância é desejável para os profissionais da saúde que trabalham no centro cirúrgico e utilizam os equipamentos em questão, pois há funções que são realizadas por um único aparelho. Por exemplo, o monitor multiparamétrico possui funções (tais como: registro do sinal de Eletrocardiografia, medição da temperatura e da pressão arterial) que os oxímetros de pulso e os oxicapnógrafos não podem fornecer.

Além disso, nos dois estudos de caso, optou-se por utilizar os resultados de trabalhos anteriores sobre manutenção preventiva no HUNRP e não fazer uma 
avaliação mais criteriosa das necessidades reais de manutenção dos equipamentos médicos, para poder simplificar o processo de coleta de dados.

Assim, a metodologia proposta vai precisar de diversos aperfeiçoamentos nos métodos de cálculos dos parâmetros envolvidos na avaliação, tais como a condição do equipamento. Adicionalmente, deverá contar com a inclusão de novos parâmetros de tomada de decisão tais como: funções adicionais desejáveis do equipamento, modularidade e intercambialidade.

Apesar disto, os estudos de caso mostraram que a metodologia é de fácil aplicação e pode fornecer uma idéia inicial das necessidades clínicas dos equipamentos médicos dos diversos setores de um hospital.

\section{Conclusão}

Este estudo mostra que a metodologia apresentada pode ser aplicada em diferentes situações. Porém é importante frisar que a simples aplicação da equação não resulta numa visão realista da situação em questão. Para um resultado satisfatório é importante a análise de alguns aspectos, envolvendo o setor do equipamento e a possibilidade de existência de outros equipamentos que tenham a mesma função. Além disso, percebe-se a importância de um método de priorização entre setores no hospital, fator importante na avaliação dos locais que realmente necessitam de reposição de equipamentos.

\section{Referências}

BRASIL. Ministério da saúde. Equipamentos para Estabelecimentos Assistenciais de Saúde: Planejamento e Dimensionamento. Brasília, 1994. 239 p.

CALIL, S. J. Análise do Setor de Saúde no Brasil na Área de Equipamentos Médico-Hospitalares In: NEGRI, B.; DI GIOVANNI, G. (Org.). Brasil: Radiografia da Saúde. Campinas: UNICAMP, 2001. p.91-121.
CAPUANO, M. Technology Acquisition Strategies for Clinical Engineering. Biomed Instrum d Technol, Arlington, v.31, n.4, p.335-357, 1997.

FELIZARDO, K. R.; ITANO, M. E.; RAMÍREZ, E. F. F. Controle de Qualidade de Oxímetros de Pulso em Hospitais. In: CONGRESSO BRASILEIRO DE ENGENHARIA BIOMÉDICA,18., 2002, São José dos Campos. Anais... São José dos Campos: JAC Editora, 2002. v.2/5, p.41-45.

HAWKINS, F. G. A. Review of Issues in Hospital Technology Acquisition. J. Clin Eng, Frederick, v.17, n.1, p.35-41, 1992.

HIRAMA, R. T. et al. Método para Inspeção de Bombas Infusoras. In: CONGRESSO BRASILEIRO DE ENGENHARIA BIOMÉDICA, 18., 2002, São José dos Campos. Anais... São José dos Campos: JAC Editora, 2002. v.2/5, p.35-40.

KATZ, Z. Estudo de Metodologias Econômicas e Multiparamétricas Aplicadas à Decisão de Substituição de Equipamentos Médicos. Dissertação (Mestrado em Engenharia Elétrica) Comissão de Pós-Graduação da Faculdade de Engenharia Elétrica e de Computação da Universidade Estadual de Campinas. Campinas, 1988. $102 p$.

LEE, P. D. The Role of Appropriate Medical Technology Procurement and User Maintenance Instructions in Developing Countries. J. Clin. Eng. Frederick, v.20, n.5, p.407-413, 1995.

MÜLLER JR., E. L. ; CALIL, S. J. Support System for Planning and Medical Equipment Specification aiming Hospital Services. In: World Congress on Medical Physics and Biomedical Engineering. [s.1.: s.n.], 2000. p.163-167.

RAMÍREZ, E. F. F.; JERONYMO NETO, O.; JANNANI, J. Método para avaliação de Propostas de Compra de Equipamentos Médicos. Semina: Ci. Exatas Tecnol., Londrina, v.22, p.3-11, 2001.

STIEFEL, R.; RISKALLA, E. The Elements of a Complete Product Evaluation. Biom. Instrum. Technol., Arlington, v.29, n.6, p.482-488, 1994.

TAWFIK, B. Evaluating Medical Equipment Needs: a Simple Algorithm. Biom. Instrum. Techno., Arlington v.28, n.3, p.187-194, 1994.

WANG, B.; CALIL, S. J. Clinical Engineering in Brazil: Current Status. J. Clin. Eng., Frederick, v.16, n.2, p.129$135,1991$. 